\title{
Analytic-Numerical Modeling and Investigation of Nanostructures' Dynamics on Material Surfaces After Laser Irradiation
}

\author{
Uldis Žaimis ${ }^{1, ~ a}$, Sharif E. Guseynov ${ }^{1,2, b}$ \\ ${ }^{I}$ Institute of Mathematical Sciences and Information Technologies, \\ Liepaja University, 14 Liela Street, Liepaja LV-3401, Latvia; \\ ${ }^{2}$ Institute of Aeronautics, Faculty of Transport and Mechanical Engineering, \\ Riga Technical University, \\ 1 A Lomonosov Street, Building-1, Riga LV-1019, Latvia \\ auldiss.z@inbox.lv, bSh.E.Guseinov@inbox.lv
}

\begin{abstract}
The work is devoted to modeling the formation and behavior of solid nano-sized particles on the surface of materials. In the simulation, it is assumed that the main processing technology of surface nanostructures is laser irradiation, which causes the Brownian motion of nanoparticles, due mainly to thermal fluctuations: if the temperature around the nanoparticles is uniformly distributed, the time average of the Brownian fluctuations is zero; however, if there is a temperature gradient around the nanoparticles, the thermal fluctuations affect the nanoparticle in different ways from different sides, and there is a force like the thermophoretic force, biasing the average position of the nanoparticle. When building a 1D model of the formation and flow behavior of nanoparticles, three important assumptions are introduced: the impact of nanoparticles on the process of irradiation is negligible; the impact of nanoparticles on each other as compared to the effect of laser irradiation on them is also negligible; and nanoparticles after laser irradiation can move both forward and backward and at every fixed period of time, moving the nanoparticles does not impose any steric constraints. Under the above assumptions, a $1 \mathrm{D}$ continuous model is built, implicit and explicit finite difference schemes to solve it are developed; their convergence and order of convergence are studied; an output condition ensuring the stability of the explicit difference scheme is obtained, the unconditional stability of implicit difference scheme is proven, and software for computer implementation of some of the obtained analytical and numerical results developed.
\end{abstract}

Keywords: Surface nanostructures, laser irradiation, mathematical modeling, single-phase model, explicit and implicit finite difference schemes

\section{INTRODUCTION}

Two of the most important trends in the field of modern nanotechnology are the development of new high-precision, low-cost methods for the synthesis of nanostructures in terms of dissipative systems and the development of decision analytical models that reflect the fundamental properties of such systems and processes. Self-organized formation of nanostructures (e.g., surface nanostructures in semiconductors) is possible in the given circumstances and supported from outside the dissipative thermodynamic process (for example, molecular beam epitaxy). Spontaneous surface nanostructures (regularly arranged pores, cracks, "shoots," protruding islands, pyramidal or conical shapes, and the like) were also observed after optical laser irradiation [1]. This method of thermal effect on the surface of some semiconductor materials has the fundamental properties of dissipative methods of the synthesis of nanomaterials. The following points are important:

1. In all cases of the synthesis, the self-organization (self-assembly) of atoms (molecules) in a regular structure is observed and due to various factors including the instant phase transition under conditions of extreme temperature and the grouping of impurity atoms in the liquid phase at the surface of the plate.

2. Self-organized structures exhibit all the signs of the nano-objects in mind, registering a quantum size effect in all cases of synthesis.

3. The system of irradiation synthesis, in our opinion, has the highest order of self- 
organization, other than the epitaxial growth system, since it "rights" chemical composition, thickness and the pattern of each layer defined in-system, and it cannot be programmed from the outside at each step.

However, the fundamental limitation of laser irradiation to date is the lack of developed techniques of controlled fusion. In other words, as a result of nanorelief, laser radiation is generated in an unpredictable manner. Based on the data input process (type of material, shape and intensity of the laser pulse and the initial temperature of the material), the nature of the future "picture" cannot be rightly determine. It should also be noted that the effective search control mechanisms of irradiation synthesis are very difficult in the absence of analytical and computational models that describe the key structural properties of the dissipative system.

\section{CONSTRUCTIONS OF MATHEMATICAL MODELS}

\section{A. Analytical model with the Dirichlet boundary} conditions, and analysis of model

Consider the following 1D quasi-linear model:

$$
\left\{\begin{array}{l}
u_{t}(x, t)+\varepsilon \cdot u_{t t}(x, t)=\nabla(k(u(x, t)) \cdot \nabla u(x, t))+f(x, t), \\
0<x<l, 0<t \leq T<\infty, \\
\left\{\begin{array}{l}
\left.u(x, t)\right|_{t=0+0}=u_{0}(x), 0 \leq x \leq l, \\
\left.u_{t}(x, t)\right|_{t=0+0}=u_{1}(x), 0 \leq x \leq l,
\end{array}\right. \\
\left\{\begin{array}{l}
\left.u(x, t)\right|_{x=0+0}=u_{2}(t), 0 \leq t \leq T, \\
\left.u(x, t)\right|_{x=l-0}=u_{3}(t), 0 \leq t \leq T,
\end{array}\right.
\end{array}\right.
$$

where $0<k(u(x, t)) \in C^{1}\{(0, l) \times(0, T]\} ;$

$0<f(x, t) \in C\{(0, l) \times(0, T]\} ;$ $u_{0}(x) \in C[0, l]$; $u_{1}(x) \in C[0, l] ; \quad u_{2}(t) \in C[0, T] ; \quad u_{3}(t) \in C[0, T]$ are given functions of their arguments; $\varepsilon, l$ and $T$ are constants. For $0<T \equiv$ const. $<\infty$, there is a time during which we investigate the dynamics of solid particles on the surface of a nano-sized material after laser irradiation. The first equation in (1) describes the temperature field of a physical system consisting of a one-dimensional (in general, non-homogeneous) material rod of length 1 . The physical meanings of the other model variables are:

- $k(u(x, t))$ is the thermal conductivity of the material rod;

- $0<\varepsilon \equiv$ const. $\ll 1$ is a control parameter of the model.

The coefficient of thermal conductivity $k(u(x, t))$

has positive temperature dependence, thus creating a positive feedback as a thermodynamic system under consideration (the so-called blow-up regime). To simplify the analysis, we can fix it as a constant, $k \equiv$ const. $>0$, or a simpler function such as, $k(u(x, t))=k(x)>0 \forall x \in[0, l]$. Mathematical models often use similar systems of the indicated ratios if the value is changed in a relatively small range. In this case we have:

$$
\left\{\begin{array}{l}
u_{t}(x, t)+\varepsilon \cdot u_{t t}(x, t)=\nabla(k(x) \cdot \nabla u(x, t))+f(x, t), \\
0<x<l, 0<t \leq T<\infty \\
\left\{\begin{array}{l}
\left.u(x, t)\right|_{t=0+0}=u_{0}(x), 0 \leq x \leq l, \\
\left.u_{t}(x, t)\right|_{t=0+0}=u_{1}(x), 0 \leq x \leq l,
\end{array}\right. \\
\left\{\begin{array}{l}
\left.u(x, t)\right|_{x=0+0}=u_{2}(t), 0 \leq t \leq T, \\
\left.u(x, t)\right|_{x=l-0}=u_{3}(t), 0 \leq t \leq T,
\end{array}\right.
\end{array}\right.
$$

where $u_{0}(x) \in C[0, l] ; \quad u_{1}(x) \in C[0, l] ; \quad u_{2}(t) \in C[0, T]$; $u_{3}(t) \in C[0, T] ; \quad 0<k(u(x, t)) \in C^{1}\{(0, l) \times(0, T]\} \quad$ are given functions of their arguments; $\varepsilon, l$ and $T$ are constants. In the proposed analytical model, the control parameter $\varepsilon$ is the most interesting option from the perspective of current research in the field of nanostructure formation during irradiation. There is a hypothesis that the value $\varepsilon$ depends on the irradiation mode (the frequency, power and pulse shape of the laser) and the physiochemical parameters of the irradiated material. In general, it is an unknown function of some physical and chemical quantities. Varying $\varepsilon$ during the numerical experiment, we can indirectly simulate the formation of nanostructures in various conditions whose presence in the analytical model is not yet possible to specify in any explicit analytical way.

Thus, the $1 \mathrm{D}$ equation in the model is more general than the classical equation of mass and heat transfer and diffusion considered, for example, in [2]. The opportunity to study the processes of formation of nanostructures in a numerical experiment which more realistically reproduces the conditions of laboratory experience is the motivation for the inclusion of the term $\varepsilon \cdot u_{t t}(x, t)$ in the model.

Let us analyze the dissipative properties of the model (1). Differential equation models include $f(x, t)>0$ and therefore characterize the open thermodynamic system. It can be argued that the term $u_{t}$ simulates the infinite growth of the temperature field in this system, "creating" extreme temperature conditions - a necessary condition for the instantaneous phase transitions with an increase in surface nanostructures. The term $\varepsilon \cdot u_{t t}(x, t)$ describes the process of decay, the scattering of thermal energy in the system, preventing it from overheating and thus its destruction. The expression $u_{t t}(x, t)$ can be interpreted as a heat wave. The control parameter $\varepsilon$ must cause asymmetry in the decision.

\section{B. Model with the Neumann boundary conditions}

The boundary conditions in the proposed model (1) and (2) are the Dirichlet boundary conditions, i.e., 
boundary conditions of the first type. The potential problem of the boundary conditions is due to the fact that the collection of the required source data may be problematic with the lack of electronic measuring instruments for recording the speed of flow of nanoparticles; $\operatorname{grad}(u(x, t))$. Dirichlet boundary conditions for these models require raw data in the form of the quantity and not the flow of the nanoparticles. In the case where the problem of recording the velocity of the flow is technically easy to implement, it is guided by the methods described, for example, in [3], as boundary conditions are preferable to be set by the Neumann condition (i.e., the boundary conditions of the second type), rather than the Dirichlet conditions. In this case, we could consider the models (1) and (2) with Neumann boundary conditions:

$$
\left\{\begin{array}{l}
u_{t}(x, t)+\varepsilon \cdot u_{t t}(x, t)=\nabla(k(u(x, t)) \cdot \nabla u(x, t))+f(x, t), \\
0<x<l, 0<t \leq T<\infty, \\
\left\{\begin{array}{l}
\left.u(x, t)\right|_{t=0+0}=u_{0}(x), 0 \leq x \leq l, \\
\left.u_{t}(x, t)\right|_{t=0+0}=u_{1}(x), 0 \leq x \leq l,
\end{array}\right. \\
\left\{\begin{array}{l}
\left.u_{x}(x, t)\right|_{x=0+0}=u_{2}(t), 0 \leq t \leq T, \\
\left.u_{x}(x, t)\right|_{x=l-0}=u_{3}(t), 0 \leq t \leq T,
\end{array}\right.
\end{array}\right.
$$

where $0<k(u(x, t)) \in C^{1}\{(0, l) \times(0, T]\} ; \quad u_{0}(x) \in C[0, l]$; $u_{1}(x) \in C[0, l] ; \quad u_{2}(t) \in C[0, T] ; \quad u_{3}(t) \in C[0, T] ;$ $0<f(x, t) \in C\{(0, l) \times(0, T]\}$ are given functions of their arguments and $\varepsilon, l$ and $T$ are constants. In the case of $k(u(x, t))=k(x)>0 \forall x \in[0, l]$, we do the same as in (2).

\section{CONSTRUCTION OF THE DISCRETE MODELS AND ANALYSIS}

\section{A. Development and investigate of explicit} difference scheme

We construct stepped grid for spatial variable $x \in[0, l]:$

$$
\begin{aligned}
& \Omega_{x}^{(1)} \stackrel{\text { def }}{\equiv}\left\{x_{j}: x_{j}=j \cdot h_{x}, 0 \leq j \leq N, h_{x}=\frac{l}{N}, N \in \mathbb{N}\right\}, \\
& \Omega_{x}^{(2)} \stackrel{\text { def }}{=}\left\{x_{j+\frac{1}{2}}: x_{j+\frac{1}{2}}=\left(j+\frac{1}{2}\right) h_{x}, 0 \leq j<N, h_{x}=\frac{l}{N}, N \in \mathbb{N}\right\} .
\end{aligned}
$$

Next, we construct regular grid for time variable $t \in[0, T]:$

$$
\Omega_{t} \stackrel{\text { def }}{\equiv}\left\{t^{n}: t^{n}=n \cdot h_{t}, 0 \leq n \leq M, h_{t}=\frac{T}{M}, M \in \mathbb{N}\right\} .
$$

Now entering the discrete function $U_{j}^{n}=u\left(x_{j}, t^{n}\right)$, we can approximate the model (2) in a discrete area in the following specific manner:

$$
\begin{aligned}
& \frac{U_{j}^{n+1}-U_{j}^{n}}{h_{t}}=-\frac{V_{j+\frac{1}{2}}^{n+1}-V_{j-\frac{1}{2}}^{n+1}}{h_{x}}+F_{j}^{n}, \\
& j=\overline{1,(N-1)}, n=\overline{0, M} ; \\
& \varepsilon \cdot \frac{V_{j+\frac{1}{2}}^{n+1}-V_{j-\frac{1}{2}}^{n}}{h_{t}}+V_{j+\frac{1}{2}}^{n+1}=-k_{j+\frac{1}{2}}^{n} \cdot \frac{U_{j+1}^{n}-U_{j}^{n}}{h_{x}}, \\
& j=\overline{1,(N-1)}, n=\overline{0, M} \text {; } \\
& \left\{U_{j}^{0}=u_{0, j}, \quad \forall j=\overline{0, N},\right. \\
& \left\{U_{j}^{1}=h_{t} \cdot u_{1, j}+u_{0, j}, \quad \forall j=\overline{0, N} ;\right. \\
& \begin{cases}U_{0}^{n}=u_{2}^{n}, & \forall n=\overline{0, M}, \\
U_{N}^{n}=u_{3}^{n}, & \forall n=\overline{0, M},\end{cases}
\end{aligned}
$$

where $V_{i}^{m}=\left.k\left(x_{i}\right) \cdot \frac{\partial u\left(x, t^{m}\right)}{\partial x}\right|_{x=x_{i}}$, i.e. $V_{i}^{m}$ is an approximation of the function $k(x) \cdot \frac{\partial u(x, t)}{\partial x}$; $F_{j}^{n}=f\left(x_{j}, t^{n}\right)$.

If it is possible to use the second type of boundary conditions we could consider the model (2) with the boundary conditions of the second kind (i.e., the Neumann conditions):

$$
\left\{\begin{array}{l}
\left.\frac{\partial \mathrm{u}(\mathrm{x}, \mathrm{t})}{\partial \mathrm{x}}\right|_{\mathrm{x}=0}=\mathrm{u}_{2}(\mathrm{t}), \quad 0 \leq \mathrm{t} \leq \mathrm{T}, \\
\left.\frac{\partial \mathrm{u}(\mathrm{x}, \mathrm{t})}{\partial \mathrm{t}}\right|_{\mathrm{x}=1}=\mathrm{u}_{3}(\mathrm{t}), \quad 0 \leq \mathrm{t} \leq \mathrm{T} .
\end{array}\right.
$$

For this case an approximation is of the form:

$$
\begin{cases}\frac{h_{x}}{2} \times \stackrel{o}{U}_{t 0}+\stackrel{o}{U}_{0}^{n+1}=u_{2}^{n}, & n=0,1, \ldots, M, \\ \frac{h_{x}}{2} \times \stackrel{o}{U}_{t N}+\stackrel{o}{U}_{N}^{n+1}=u_{3}^{n}, & n=0,1, \ldots, M,\end{cases}
$$

where we have the following notation:

$$
\begin{cases}\stackrel{\circ}{U}_{t a} \stackrel{\text { def }}{\equiv} \frac{U_{j(=a)}^{n+1}-U_{j(=a)}^{n}}{h_{t}} & (a=\{0 ; N\}), \\ \stackrel{\circ}{U}_{a} \stackrel{\operatorname{def}}{\equiv} \frac{U_{j(=a)+1}^{n+1}-U_{j(=a)}^{n+1}}{h_{x}} & (a=\{0 ; N\}) .\end{cases}
$$

We return to the difference scheme (4)-(7). Substituting (5) into (4) and using the new designations

$$
\begin{gathered}
U_{t} \stackrel{\operatorname{def}}{\equiv} \frac{U_{j}^{n+1}-U_{j}^{n}}{h_{t}} ; \\
U_{\bar{t}} \stackrel{\operatorname{def}}{\equiv} \frac{U_{j}^{n}-U_{j}^{n-1}}{h_{t}} ; \\
U_{\bar{x}} \stackrel{\operatorname{def}}{\equiv} \frac{U_{j}-U_{j-1}}{h_{x}} ;
\end{gathered}
$$




$$
U_{x} \stackrel{\text { def }}{=} \frac{U_{j+1}-U_{j}}{h_{x}},
$$

we obtain the following explicit finite difference scheme for the mathematical model (2):

$$
\left\{\begin{array}{l}
\varepsilon \cdot U_{\overline{t t}}^{n}+U_{t}^{n}=\partial_{x}\left(k \cdot \partial_{\bar{x}} U^{n}\right)+F_{j}^{n}, \quad j=1,2, \ldots, N-1 ; \\
\left\{\begin{array}{l}
U_{j}^{0}=u_{0, j}, \quad j=1,2, \ldots, N ; \\
U_{j}^{1}=h_{t} \cdot u_{1, j}+u_{0, j}, \quad j=1,2, \ldots, N ;
\end{array}\right. \\
\begin{cases}U_{0}^{n}=u_{2}^{n}, \quad n=0,1, \ldots, M ; \\
U_{N}^{n}=u_{3}^{n}, \quad n=0,1, \ldots, M\end{cases}
\end{array}\right.
$$

The cconvergence and stability of the scheme are not presented here due to limited space.

\section{B. Development and investigate of implicit difference scheme}

In this section, an implicit difference scheme is developed on a discrete area $\Omega_{t} \times\left\{\Omega_{x}^{(1)} \times \Omega_{x}^{(2)}\right\}$ for the approximation of the mathematical model (2), where nets $\Omega_{t}, \quad \Omega_{x}^{(1)}$ and $\Omega_{x}^{(2)}$ are constructed as in the previous section:

$$
\begin{aligned}
& \Omega_{x}^{(1)} \stackrel{\text { def }}{\equiv}\left\{x_{j}: x_{j}=j \cdot h_{x}, 0 \leq j \leq N, h_{x}=\frac{l}{N}, N \in \mathbb{N}\right\} ; \\
& \Omega_{x}^{(2)} \stackrel{\text { def }}{=}\left\{x_{j+\frac{1}{2}}: x_{j+\frac{1}{2}}=\left(j+\frac{1}{2}\right) h_{x}, 0 \leq j<N, h_{x}=\frac{l}{N}, N \in \mathbb{N}\right\} ; \\
& \Omega_{t} \stackrel{\text { def }}{\equiv}\left\{t^{n}: t^{n}=n \cdot h_{t}, 0 \leq n \leq M, h_{t}=\frac{T}{M}, M \in \mathbb{N}\right\} .
\end{aligned}
$$

As in Section 3.1, where we approximate mathematical model (2), we obtain:

$$
\begin{aligned}
& \frac{U_{j}^{n+1}-U_{j}^{n}}{h_{t}}=-\frac{V_{j+\frac{1}{2}}^{n+1}-V_{j-\frac{1}{2}}^{n+1}}{h_{x}}, \\
& \forall j=\overline{1,(N-1)} ; \quad \forall n=\overline{0, M} ; \\
& \varepsilon \frac{V_{j+\frac{1}{2}}^{n+1}-V^{n}}{h_{t}}+V_{j+\frac{1}{2}}^{n+1}=-k_{j+\frac{1}{2}}^{n+1} \frac{U_{j+\frac{1}{2}}^{n+1}-U_{j}^{n+1}}{h_{x}}+F_{j}^{n}, \\
& \left\{\begin{array}{l}
U_{j}^{0}=u_{0, j}, \quad \forall j=\overline{0, N}, \\
U_{j}^{1}=h_{t} \cdot u_{1, j}+u_{0, j}, \quad \forall j=\overline{0, N} ;
\end{array}\right. \\
& \left\{\begin{array}{l}
U_{0}^{n}=u_{2}^{n}, \quad \forall n=\overline{0, M}, \\
U_{N}^{n}=u_{3}^{n}, \quad \forall n=\overline{0, M},
\end{array}\right.
\end{aligned}
$$

Substituting (9) with (11) into (10), we obtain the following implicit, finite difference scheme approximating the continuous mathematical model (2):

$$
\left\{\begin{array}{l}
\varepsilon \cdot U_{\overline{t t}}^{n}+U_{t}^{n}=\partial_{x}\left(k \cdot \partial_{\bar{x}} U^{n+1}\right), \\
\forall j=1,2, \ldots, N-1 ; \\
\left\{\begin{array}{l}
U_{j}^{0}=u_{0, j}, \quad j=0,1, \ldots, N ; \\
U_{j}^{1}=h_{t} \cdot u_{1, j}+u_{0, j}, \quad j=0,1, \ldots, N ;
\end{array}\right. \\
\left\{\begin{array}{l}
U_{0}^{n}=u_{2}^{n}, \quad n=0,1, \ldots, M ; \\
U_{N}^{n}=u_{3}^{n}, \quad n=0,1, \ldots, M .
\end{array}\right.
\end{array}\right.
$$

C. The main properties of the constructed difference schemes

- The developed explicit difference scheme (4) (7), which approximates the mathematical model (2), is easier to implement with a computer than the developed implicit difference scheme (9) (12) approximating the same mathematical model;

- The order of convergence of the two difference schemes is the same. The two difference schemes' estimate in the energy norm is $\left\|u\left(t^{n}\right)-U^{n}\right\|_{\infty} \leq$ Const $\cdot\left(h_{t}+h_{x}^{2}\right)$, for each of which is developed its own Const and only these constants are different, not the orders of convergence;

- The explicit difference scheme (4) - (7) is a conditionally stable difference scheme, and for its stability it is sufficient to choose the steps $h_{x}$ and $h_{t}$, respectively of a spatial grid $\Omega_{x}^{(1)}$ and regular grid $\Omega_{t}$ such that:

- when $k(x) \equiv$ const, $\forall x \in(0, l)$, the inequality $\quad\left\|u\left(t^{n}\right)-U^{n}\right\|_{\infty} \leq \bar{C} \cdot\left(h_{t}+h_{x}^{2}\right)$, called a sufficient condition for stability of (4) - (7) holds, where $\bar{C}$ is some constant, and $\|\cdot\|_{\infty}$ is the energy norm, determinated as $\|A\|_{\infty} \stackrel{\text { def }}{\equiv} \max _{0 \leq j \leq N}\left|A_{j}\right|$;

- when $k(x) \not \equiv$ const, $\forall x \in(0, l)$, the inequality $B>\frac{1}{4} \cdot C$ holds, where

$$
B \stackrel{\operatorname{def}}{\equiv} \frac{\varepsilon+\frac{h_{t}}{2}}{h_{t}^{2}} \cdot E \text { and } C U^{n} \stackrel{\text { def }}{\equiv}-\partial_{x}\left(k \cdot \partial_{\bar{x}} U^{n}\right) ;
$$

- The implicit difference scheme (9) - (12) is an unconditionally stable difference scheme for the first case when $k(x) \equiv$ const, $\forall x \in(0, l)$, and for the second case, when $k(x) \not$ const, $\forall x \in(0, l)$.

Otherwise, for the computer implementation of the implicit difference scheme (9) - (12), steps $h_{x}$ and $h_{t}$ 
respectively of a spatial grid $\Omega_{x}^{(1)}$ and regular grid $\Omega_{r}$ can be chosen arbitrarily, guided only by the consideration of the size of $h_{t}+h_{x}^{2}$ and without fear of problems in the theory of difference schemes (in general and in the theory of numerical methods) or the volatility of the developed methods with respect to the original initial and boundary data.

\section{NUMERICAL EXPERIMENTS}

The theory outlined above, based on an explicit difference scheme, is a computer program written in the software MatLAB for numerical experiments. Using coefficients $\varepsilon=1$ and $k=0.3$ and initiating systems with short-term external influence at time $t=0.04$, the outcome of Fig. 1 is obtained, which shows the projection of the material over time. In this example we used the Dirichlet boundary conditions.

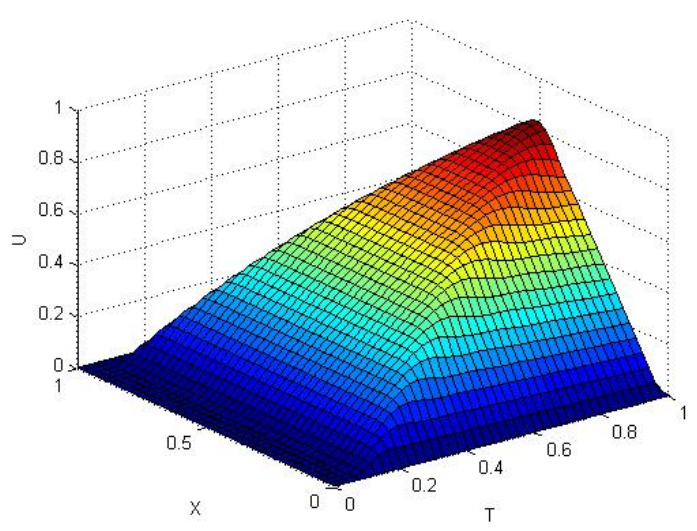

Fig. 1. Illustration of the process of forming a nanoscale spire: realization of the discrete model (4)-(7) under $\varepsilon=1$ ("bad case").

\section{$\mathrm{V}$ CONCLUSIONS}

This work concentrated on developing a model of the effects of solid nanoparticles on the surface of materials. The 1D continuous analytical model was constructed with both Neumann and Dirihlet boundary conditions. Both implicit and explicit finite difference schemes were constructed and their convergence and stability investigated. Finally, an example of a numerical experiment was done with computer software developed form the theory.

\section{ACKNOWLEDGMENTS}

For the second co-authors the present article was executed within the framework of the following two Research Projects: 1) The State Research Programme "NextIT": Next generation Information and Communication Technologies; 2) The European Regional Development Fund Project No. 2014 / 0029 / 2DP / 2.1.1.1 / 14 / APIA / VIAA / 088: "Development of an experimental long flight distance unmanned aerial vehicle prototype for multi-purpose environmental monitoring".

\section{REFERENCES}

[1] A. Medvid, Nano-cones Formed on a Surface of Semiconductors by Laser Radiation: Technology, Model and Properties, Proceedings of the International Conferences on Nanowires Science and Technology, February 2010. Croatia: INTECH. pp. 402-424.

[2] J. Kaupuzs, Sh. E. Guseynov, J. S. Rimshans, and A. Medvid, "Modeling of surface structure formation after laser irradiation." International Journal of Recent Advances in Fluid Mechanics, Heat \& Mass Transfer and Biology. pp. 49-56. 2011.

[3] A. N. Tikhonov, and A. A. Samarsky, Equations of Mathematical Physics. Moscow: Lomonosov Moscow State University Publishing, 1999. 